In der SÄZ 4/2012 erschien die Zusammenfassung einer Studie zur Kosteneffizienz von Ärztenetzwerken [1], die mittelfristig erhebliche Einsparungen in Managed-Care-Modellen nachwies. Die Ergebnisse dieser Studie wurden in der SÄZ 17/2012 als nicht signifikant kritisiert [2] - ein aus Sicht der involvierten Wissenschaftler unhaltbarer Vorwurf. Anstatt sich in Re- und Dupliken einen Schlagabtausch zu liefern, suchten die Autoren der beiden Artikel das Gespräch, dessen Ergebnis wir in Form des nachfolgenden Beitrags der Leserschaft gerne präsentieren.

Die Redaktion

\title{
Kosteneinsparungen mit Managed Care: Diskussion um Signifikanz von Studienresultaten
}

René Haldemann

* Laurence Sterne (1713-1768), englischer Schriftsteller

Korrespondenz:

Dr. med. René Haldemann

Gartenstrasse 8

CH-8805 Richterswil

Tel. 0438889575

Fax 0438889576

rene.haldemann[at]hin.ch

\section{Einleitung}

In meiner Publikation mit dem Titel «Bringt Budgetmedizin doch keine Einsparungen?», die als Standpunkt in Ausgabe 17 der Schweizerischen Ärztezeitung am 25.4.2012 erschienen ist [2], wurde die ökonomische Studie von Maria Trottmann, Konstantin Beck und Ute Kunze unter dem Titel «Steigern Schweizer Ärztenetzwerke die Effizienz im Gesundheitswesen?» (erschienen in der SÄZ 4/2012) kritisch beleuchtet. In der Tabelle 3 zur Studie wurden die Ergebnisse für die drei Analysemethoden mit jeweils drei statischen Masszahlen präsentiert. Da weder Konfidenzintervalle noch statistische Signifikanzen angegeben wurden, habe ich in der Annahme, dass es sich bei der Masszahl der Streuung um den gepoolten Standardfehler handelt, die Schlussfolgerung gezogen, dass die Einsparungen statistisch nicht signifikant sein können.

Erst durch die ergänzenden Erläuterungen in der nachfolgenden Präzisierung von Professor Beck wird ersichtlich, dass es sich um ein $6 \times 4$ Blockdesign handelt, wobei die Stichproben nicht unabhängig sind, was die statistische Bewertung ohne nähere Angaben zur Methodik erschwert. Retrospektiv gesehen, war meine Schlussfolgerung bezüglich der Signifikanz de facto voreilig, und eine vorgängige Kontaktaufnahme mit den Autoren der Studie wäre sinnvoll gewesen. An den übrigen Aussagen halte ich fest, insbesondere was die externe Validität, d. h. die Übertragbarkeit der Ergebnisse bei flächendeckender Einführung der Budgetverantwortung, angeht, da ein ausgeprägter Selektionseffekt für diese Versicherungsform besteht.

Professor Beck und ich haben es vorgezogen, die Auseinandersetzung bezüglich der Signifikanz der Studienergebnisse in einem gemeinsamen Artikel in konstruktiver Art zu vollziehen, statt eines Schlagabtausches in Form von Repliken und Dupliken. Wir wollen auf dem Schlachtfeld der Zahlen keine Verlierer. Wir möchten damit auch ein Zeichen setzen, sich mit gegenseitigem Respekt zu begegnen.

«Respect for ourselves guides our morals; respect for others guides our manners.» *

\section{Zur Frage der Einsparungen in Managed- Care-Modellen: eine Präzisierung unserer Forschungsresultate}

Konstantin Beck ${ }^{a, b}$, Ute Kunzea , Maria Trottmann $^{b}$, Monika Buholzer ${ }^{a}$

a CSS Institut für empirische Gesundheitsökonomie

b Institut für Volkswirtschaftslehre, Universität Zürich
Wir danken Herrn Dr. Haldemann für seine einleitenden Worte und wollen uns im Folgenden auf die technischen Aspekte der Studie konzentrieren, die in unserem ursprünglichen Beitrag [1] nicht ausreichend präzise herausgearbeitet worden sind. In seinem Artikel [2] in der Schweizerischen Ärztezeitung vom 25.4.2012 kritisiert Dr. Haldemann unsere Studie zur Kosteneinsparung ([1] respektive [3]). Er versucht dabei, den Nachweis zu erbringen, dass die ausgewiesenen Kostenvorteile von Managed-CareModellen nicht signifikant seien. Schon seit Wo- chen wundern wir uns über die im Abstimmungskampf oft wiederholte Behauptung, es gäbe keine einzige Studie, die den Nachweis signifikanter Kosteneinsparungen bei Managed-Care-Modellen erbringe. Dank des Beitrags von Dr. Haldemann können wir aufzeigen, woher diese Vorstellung rührt.

Seine Argumentation basiert grob umrissen auf Tabelle 3 [1], in der eine mittlere Einsparung von $11,9 \%$ zusammen mit einer Standardabweichung von $8,6 \%$ angegeben wird (CART-Methode). Mittelwert und Standardabweichung beziehen sich auf 


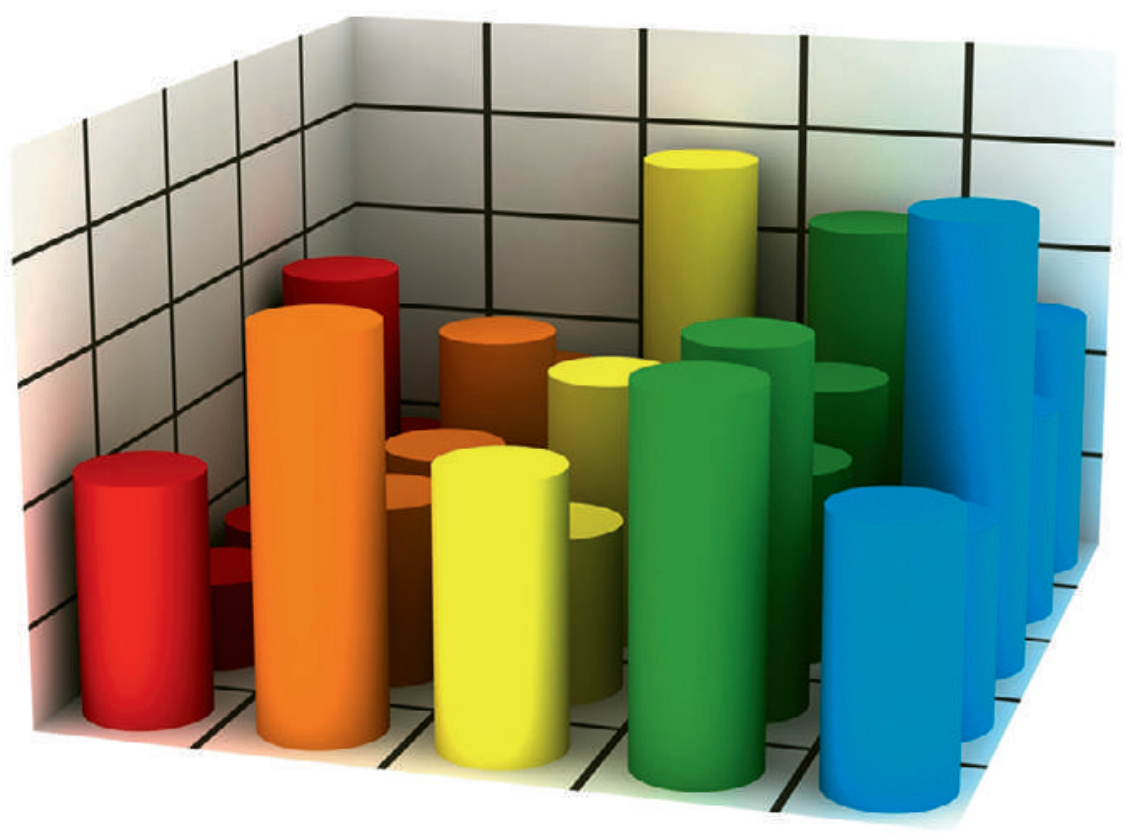

Auch Statistiken, sobald ungünstig präsentiert, können den Blick auf die zugrundeliegenden Zusammenhänge verstellen.
Warum handelt es sich aber bis hierher lediglich um eine scheinbare Signifikanz? Auch unter Verwendung der Wurzelfunktion ist die so durchgeführte Signifikanzanalyse problematisch, weil es sich dabei um 24 Mittelwerte handelt, die auch einzeln mit Varianz behaftet und zudem nicht unabhängig voneinander sind. Je sechs beziehen sich auf dasselbe Jahr und wiederum je vier auf dasselbe Ärztenetzwerk. In einer früheren Arbeit [4] hatten wir daher die Signifikanz der einzelnen Resultate mittels Bootstrap geprüft, was wir nun auch für die aktuellsten Daten wiederholten. Für die sechs untersuchten Ärztenetzwerke, das Jahr 2009 und die Zwillingsmethode ergeben sich stets signifikante Einsparungen. Auch wenn wir aus Zeitgründen nicht sämtliche 72 Varianten im Detail nachprüfen konnten - in drei Fällen traten negative Einsparungen auf, so dass positive Signifikanz offensichtlich nicht gegeben ist reicht dieser Befund für die Aussage, dass signifikante Einsparungen in Managed-Care-Modellen gegeben sind. Das ist nicht zu verwechseln mit der Aussage, dass sämtliche Einsparungen in sämtlichen existierenden Modellen signifikant positiv ausfallen würden.

Mit dieser Schlussfolgerung sind wir nicht allein. Allein von den hier Schreibenden gibt es zwei weitere Publikationen, in denen signifikante Einsparungen von Managed-Care-Modellen nachgewiesen werden $[4,5]$. Darüber hinaus existieren vier wissenschaftliche Untersuchungen von anderen Autoren, die unseren Befund bestätigen [6-9], sowie eine ältere Untersuchung [10], bei der Einsparungen errechnet werden, deren Signifikanz jedoch nicht über jeden Zweifel erhaben nachgewiesen werden kann. Uns ist somit keine einzige jüngere Untersuchung zu diesem Thema bekannt, die nicht signifikante Kostenvorteile zutage gefördert hätte.

Zerechnung der Signifikanz ist nicht die dardabweichung der Mittelwerte, sondern die Standardabweichung, dividiert durch die Wurzel der Anzahl Beobachtungen (hier 24) minus eins gemäss dem Gesetz der grossen Zahl) zu verwenden. Das 95\%-Vertrauensintervall (unter Verwendung der korrekteren $t$-Verteilung) liegt dann bei der mittleren Einsparung von $11,9 \%$ zwischen $8,2 \%$ und $15,6 \%$

\section{Zum Nutzen von Managed Care}

Wie steht es nun mit der Frage des Nutzens von Bedass der Nutzen in diesen Untersuchungen nicht explizit modelliert und kontrolliert worden ist. Bei den handlungen in Managed-Care-Modellen? Richtig ist,

\section{Uns ist somit keine einzige jüngere Untersuchung zu diesem Thema bekannt, die nicht signifikante Kostenvorteile zutage gefördert hätte.}

Korrespondenz: CSS Institut für empirische Gesundheitsökonomie Tribschenstrasse 21 CH-6002 Luzern Tel. 0582771726 info.institut[at]css.ch www.css-institut.ch
$(11,9 \%+/-3,7 \%)$. Im Fall der Zwillingsmethode, mit einer mittleren Einsparung von 13,0\%, zwischen $10,1 \%$ und $15,9 \%(13,0 \%+/-2,9 \%)$. Beide mittleren Einsparungen sind somit (scheinbar) eindeutig signifikant. Zugegebenermassen war der Sachverhalt in unserer Tabelle nicht ganz so klar dargestellt, was Anlass zur irrtümlichen Signifikanzberechnung durch Dr. Haldemann gab. heutigen Managed-Care-Modellen handelt es sich um offene Systeme in Konkurrenz zur normalen Hausarztversorgung. Wenn die Versorgungsqualität nicht den Wünschen entspricht, können die Versicherten zeitnah ins traditionelle System wechseln. Die Risikostruktur dieser Wechsler wurde ebenfalls untersucht, und es zeigte sich, dass der oft gehörte Vorwurf, Managed Care sei nur gut für Gesunde, 
weil jenen die ungenügende Behandlungsqualität nicht auffallen könne, empirisch nicht gestützt werden kann. Einzig wenn unterstellt wird, dass Managed-Care-Patienten nicht in der Lage sind, eine Behandlungsqualität $\mathrm{zu}$ beurteilen, und auch bei schlechter Qualität freiwillig in einem ManagedCare-Modell verbleiben, sind unsere Resultate in Zweifel zu ziehen. Eine solche Einschätzung mag für einzelne, aber sicher nicht für das Gros der Managed-Care-Patienten zutreffend sein.

\section{Wir glauben, den Nachweis erbracht zu haben, dass Managed- Care-Modelle in der Lage sind, signifikante Einsparungen zu erbringen.}

Auch den dritten Kritikpunkt, es liege ein «Regression-to-the-mean-Effekt» vor, haben wir uns noch einmal genauer angeschaut. Wir stellen fest, dass lediglich bei 3 von 18 untersuchten Zeitreihen ein signifikant negativer Trend (d. h. ein Rückgang der Einsparungen im Laufe der Jahre) diagnostiziert werden kann, alle übrigen weisen entweder keinen oder sogar einen signifikant positiven Trend aus. «Regression to the mean» scheint demnach als Erklärung wegzufallen.

Zusammenfassend glauben wir, mit unseren Ausführungen den Nachweis erbracht zu haben, dass Managed-Care-Modelle durchaus in der Lage sind, signifikante Einsparungen zu erbringen.

\section{Literatur}

1 Trottmann M, Beck K, Kunze U. (2012). Steigern Schweizer Ärztenetzwerke die Effizienz im Gesundheitswesen? Eine Analyse auf mittlere Frist. Schweiz Ärztezeitung. 2012; 93(4):125-7.

2 Haldemann R. Bringt Budgetmedizin doch keine Einsparungen? Schweiz Ärztezeitung. 2012;93(17):636-7.

3 Beck K, Kunze U, Buholzer M, Trottmann M. Steigern Schweizer Ärztenetzwerke die Effizienz im Gesundheitswesen? Eine Analyse auf mittlere Frist. Forschungsbericht des CSS Institut für Empirische Gesundheitsökonomie. 2011. www.css-institut.ch/de/ home/wissenschaftliche_publikationen/managed_ care_und_capitation.html

4 Beck K, Käser U, Trottmann M, von Rotz S. Effizienzsteigerung dank Managed Care? Evidenz aus der Schweiz. Datamaster (Beiheft zum Clinicum). 2009:15-21.

5 Trottmann M, Zweifel P, Beck K. Supply-side and demand-side cost sharing in deregulated social health insurance: Which is more effective? Journal of Health Economics. 2012;31(1):231-42.

6 Lehmann HJ. Managed Care - Kosten senken mit alternativen Versicherungsformen? Chur/Zürich: Verlag Rüegger; 2003.

7 Lehmann HJ, Zweifel P. Innovation and Risk Selection in Deregulated Social Health Insurance. Journal of Health Economics 2004;23:997-1012.

8 Grandchamp C, Gardiol L. Does a mandatory telemedicine call prior to visiting a physician reduce costs or simply attract good risks? Health Economics. 2010; published online in Wiley Online Library, DOI $10.1002 /$ hec. 1668 .

9 Reich O, Rapold R, Flatscher-Thöni M. An empirical investigation of the efficiency effects of integrated care models in Switzerland. International Journal of Integrated Care. 2012;12:1-12.

10 Schwenkglenks M, Preiswerk G, Lehner R, Weber F, Szucs TD. Economic efficiency of gatekeeping compared to fee for service plans: a Swiss example. Journal of Epidemiology and Community Health. 1998;60:24-30. 